\title{
A 3D Serious Game for Dental Learning in Higher Education
}

\author{
David Rodríguez-Andrés; M.-Carmen Juan; Ramón Mollá \\ Instituto Universitario de Automática e Informática \\ Industrial \\ Universitat Politècnica de València \\ $\mathrm{C} /$ Camino de Vera, $\mathrm{s} / \mathrm{n}$ \\ 46022-Valencia, Spain \\ \{drodriguez, mcarmen, rmolla\}@dsic.upv.es
}

\author{
Magdalena Méndez-López \\ Departamento de Psicología y Sociología \\ Universidad de Zaragoza \\ Zaragoza, Spain \\ mmendez@unizar.es
}

\begin{abstract}
Natural User Interfaces and advanced displays can be combined to provide rich learning experiences. In this paper, we present the development and validation of a serious game that combines autostereoscopy and Natural User Interfaces for dental learning in higher education. The game includes two modes: neutral and real-world background. A comparative study to check different aspects was carried out. A total of 33 dentistry students participated in the study. From the results, the students increased significantly their knowledge about teeth morphology. Most of the students preferred the neutral background for dental learning. The real-world background was identified as being more suitable for leisure activities.
\end{abstract}

Keywords- Autostereoscopy, Natural User Interfaces, education, serious game

\section{INTRODUCTION}

The rapid development of new technologies has provided many new systems that were unimaginable just a few years ago. Natural User Interfaces (NUI) have become more and more common. They facilitate our interaction with systems without having to use extra devices; we can use some parts of our body to communicate with the systems. Another technology to consider is autostereoscopy, which is a method for displaying stereoscopic images without the use of special glasses on the part of the viewer.

Dentistry students do not usually have any 3D virtual model to help them learn about teeth morphology. They usually have real models made of plaster or 3D models drawn on paper. From our point of view, a 3D virtual model could help in this learning process. If the virtual model was also presented in 3D with autostereoscopy, the 3D visualization could further improve the learning process. If the interaction is as natural as possible (for example, using NUI), the learning process could also be more natural and intuitive. The users of this type of visualization do not need to wear or manipulate any physical device to interact with the system. The virtual elements are perceived in $3 \mathrm{D}$ as real elements and the manipulation is performed with gestures. Following this idea, we have developed a dental learning game that includes NUI and autostereoscopy. To validate the game, we measured the influence of the self-representation and the NUI. We compared the real-world background (the users can see themselves as in a mirror) with a neutral background. Our first hypothesis was that users would increase their knowledge about the teeth morphology thanks to the $3 \mathrm{D}$ visualization of the models. Our second hypothesis was that since the real-world background offers more cues about the environment than the neutral background, there would be a statistically significant improvement in knowledge in favor of the real-world background.

\section{BACKGROUND}

NUI allow users to be the controller of themselves by detecting the position of the different parts of their body. According to Fishkin [1], NUIs facilitate the acceptance of an application by users. Roman [2] pointed out "The mouse's days are numbered", the current trend in new devices, games, and consoles is to get rid of all gamepads, joysticks, and other input methods.

NUI are being incorporated in different types of applications, for example, learning [3], assessment of shortterm memory [4]; training individuals with cognitive impairments [5]; or transforming a paper based process to a NUI process in a chronic care hospital [6].

Since the publication of the first papers about stereoscopy in the 1990s [7], several works have been presented related with this technology. For example, a virtual reality immersive experience utilizing 35" LCD 3D displays that used the parallax barrier technique [8]. An application with Kinect( $\bigcirc$ Fusion that captured a 3D image of a realworld scene which was reproduced in an autostereoscopic display [9]. Kim et al. [10] used autostereoscopy combined with NUI making an autostereoscopic display for multi-user collaboration. Kim et al.'s study proposed an autostereoscopic platform for sharing visual data with two or more users, which uses two displays. Studies have also been carried out to compare augmented and virtual reality using autostereoscopic displays [11].

Several dental learning systems have been presented. However, most of them are pretty basic; some of them use web technologies [12], or multimedia material. Grigg and Stephens [13] studied the possibility to learn dentistry using computers. They evaluated the knowledge of their time in an objective manner to make some predictions such as that computer-assisted learning would have an impact not only on how dentists would be trained, but also on the skills they would need to acquire in the future in order to keep up with new technologies. Computer-aided dental learning programs 


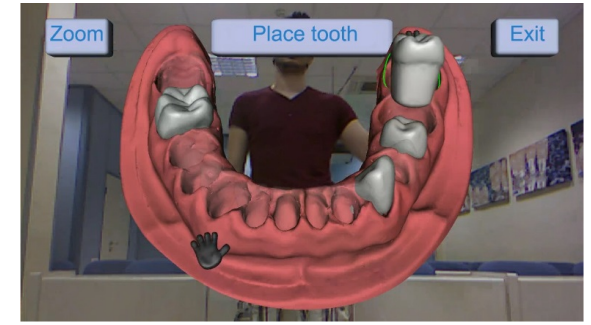

Fig. 1 Screen view of the real-background mode.

are either more effective than or equally effective as other methods of education [14]. The benefits of computer-assisted learning can be seen for example in self-paced and selfdirected learning and increased motivation [15]. The effectiveness of computer-aided programs in dental education have also been analyzed [16]. Those authors concluded that computer-assisted learning can provide innovative and interactive ways of presenting material, and, therefore, should be used with conventional teaching or as a mean of self-instruction that can elicit a positive response from students and can motivate them. Following these recommendations and unlike previous works, we propose to combine stereoscopy and NUIs for dental learning.

\section{DEVELOPMENTS}

Our game combines autostereoscopy and natural interaction. We use a Microsoft Kinect $\odot$ device to capture the image of the real world and to track the user. We use an autostereoscopic display for the 3D perception. The user does not need to wear any extra devices to interact with the game. The virtual elements are objects with 3D perception. Students are able to interact with the game using gestures.

The 3D display is on a table near a wall in front of the user. The Microsoft Kinect $C$ is placed on the table a few centimeters in front of the display and centered relative to the 3D display. The users should stand in front of the display two meters away.

We developed two different versions of the game. The functionality is the same for the two versions. The only difference is the visualization of the background. In one version (the real-world background), the real world captured by the Kinect camera is shown as the background (Fig. 1). Therefore, the users see the real world and they can also see themselves as in a mirror. In the second version (the neutral background), the background is a neutral blue color.

The main goal of the user in the two versions is to place each tooth in its correct position. In our game, seven teeth of the lower jaw have been included. Looking closely at the 3D models from all angles, the students can observe minimal details that help them to identify each tooth in order to place it in the right position. The game is divided into two principal stages (1. Learning stage and 2. Placement stage).

In the Learning stage, the user sees all of the 3D models for the teeth. The game shows and reproduces the scientific

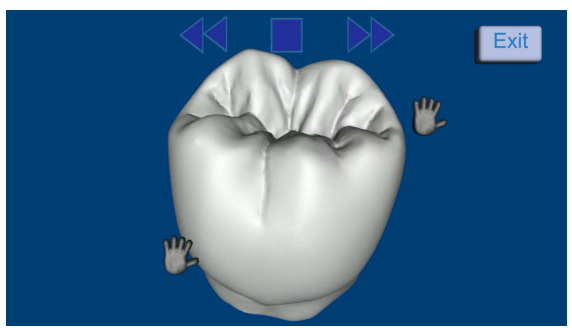

Fig. 2 Zoom of a tooth.

name of each tooth. The principal goal at this stage is for the users to observe each tooth and to learn about its morphology. The teeth are presented one after another. When all the teeth have been shown, the game changes to the next stage.

In the Placement stage, the game shows the lower jaw in the center of the screen with fourteen holes, one hole for each tooth. Three buttons appear in the upper area of the screen. When one of these buttons is selected, the related action will be carried out. Fig. 1 shows a screenshot of this stage. The three buttons are:

1) Zoom Button. When this button is selected, the game shows a detailed view of the tooth that the user is handling. The tooth is ten times bigger than the tooth visualized in the placement view. With this detailed view, the user can better appreciate the morphology of the tooth and know its correct placement in the jaw. Fig. 2 shows an example. The options that appear in the center of this view work as follows: (a) $\mathbf{K}$ : The tooth rotates counter-clockwise. (b) $\boldsymbol{~}$ : The tooth rotates clockwise. (c) $\square$ This button stops the rotation of the tooth. The "Exit button" allows the user to go back to the previous view. The selection of these buttons is similar to the previous ones. The users only have to put their hand over the button to select them.

2) Place tooth button. This button allows a selected tooth to be placed in one of the fourteen holes. To achieve this, the user has to put the hand that holds the tooth over the hole of its correct position and with the other hand select the Place tooth button.

3) The Exit button allows the user to exit the game.

\section{STUDY}

A total of 33 students from a School of Dentistry participated in our study. There were 18 men $(52.34 \%)$ and 15 women $(47.66 \%)$. They were specializing in two areas: Hygiene and Prosthesis. They were divided into two groups. Each group had participants from both specialties who were assigned to one of the following two groups:

- Group A: Participants that played with the real-world background configuration first, and afterwards played with the neutral background configuration. There were 17 participants (Hygiene $=9$; Prosthesis $=8$ ). 
- Group B: Participants that played with the neutral background configuration first, and afterwards played with the real-world background configuration. There were 16 participants $($ Hygiene $=8$; Prosthesis $=8$ ).

To collect the data, we designed three different questionnaires. The Q1 (the knowledge questionnaire) to measure knowledge about teeth morphology. In this questionnaire, the students have to connect each tooth with its correct position using arrows in a paper with the images of the teeth and the jaw. The Q1 questionnaire evaluates the knowledge about teeth morphology The Q2 questionnaire (Table I) includes questions related to usability, experienced fun, depth perception, and perceived learning. The Q2 and Q3 questionnaires were based on the questionnaire used by Martín-SanJosé et al. [3]. The final questionnaire, Q3 (Table I), which were designed to compare the two modes includes some questions about the users' preferences. In addition, the game stores information about the time users require to complete the game and the errors they have committed. The entire activity (Q1, Q2 and Q3 questionnaires and the two game modes) lasted around 30 minutes. The following protocol was used:

- The two groups filled out the Q1 questionnaire.

- Group A played with the neutral-background version and group B played with the real-world background version.

- The two groups filled out the Q1 and Q2 questionnaires. The Q1 (knowledge questionnaire) is only filled out once after playing with the first version. In other words, the analysis about knowledge outcomes is an inter-subjects analysis (Group A vs. Group B).

- Each group played the game with the mode that they had not previously played.

- The two groups filled out the Q2 and Q3 questionnaires.

\section{RESUlts}

\section{A. Knowledge Outcomes}

To measure the initial or acquired knowledge, we used the Q1 questionnaire. The knowledge score ranges from zero to seven hundred. These scores were established by an expert professor of the School of Dentistry. The knowledge variable was created to condense the correct placement of the seven teeth. Several t-tests were performed to determine if there were statistically significant differences in the acquired knowledge. All tests are shown in the format: (statistic [degrees of freedom], p-value, Cohen's d); and the "**" character indicates statistical significance at level $\alpha=0.05$. First, we checked whether there were statistically significant differences for the initial knowledge of the students in the two groups. The initial scores of knowledge for Group A (mean $420.62 \pm 134.65$ ) and for Group B (mean $380.88 \pm$ 168.8) showed that there was no statistically significant difference $(\mathrm{t}[31]=-0.72, \mathrm{p}=0.476, \mathrm{~d}=0.25)$. This result implies that there is no statistically significant difference between the two groups for the initial knowledge. For Group A, a paired t-test revealed that there was a statistically
TABLE I. Q2 AND Q3 QUESTIONNAIRES

\begin{tabular}{cl}
\hline \# & \\
\hline Q01 & How much fun did you have? \\
Q02 & $\begin{array}{l}\text { How many of your classmates would you recommend this } \\
\text { game to? }\end{array}$ \\
Q03 & What was the difficulty of the game? \\
Q04 & Did you understand the game rules? \\
Q05 & Was selecting the answers easy? \\
Q06 & How much did you like the images of the game? \\
Q07 & How much did you learn during the game? \\
Q08 & How often did you feel that the 3D models of the game \\
& looked like plaster models? \\
Q09 & Rate the sensation of viewing the teeth. Did they look like \\
& coming out of the screen? \\
Q10 & Did you have the feeling that you can touch the teeth? \\
Q11 & Rate the experience from 1 to 10. \\
\hline Q12 & Which game did you like the most? \\
Q13 & Which game is better for dental learning? \\
Q14 & Which game was easier to interact with? \\
Q15 & Which game was more comfortable? \\
Q16 & Which game did you have more control of? \\
Q17 & In which game did the images look better in? \\
Q18 & Which game was easier to interact with? \\
Q19 & Which game was more fun? \\
Q20 & Which game do you want your teacher to use in class? \\
\hline \hline The Q2 questionnaire includes Q1-Q11 questions The Q3 questionnaire includes Q12-
\end{tabular}
Q20 questions.

TABLE II. MANN WHITNEY U TEST FOR Q2 QUESTIONS

\begin{tabular}{|c|c|c|c|c|c|c|c|c|c|}
\hline \# & \multicolumn{2}{|l|}{ Mean GA } & \multicolumn{2}{|c|}{ Mean GB } & \multicolumn{2}{|c|}{$\mathrm{U}$} & Z & $\mathrm{p}$ & $\mathrm{r}$ \\
\hline Q01 & \multicolumn{2}{|l|}{$4.12 \pm 0.68$} & \multicolumn{2}{|c|}{$4.00 \pm 0.71$} & \multicolumn{2}{|c|}{148.0} & 0.47 & 0.773 & 0.082 \\
\hline Q02 & \multicolumn{2}{|c|}{$4.41 \pm 0.60$} & \multicolumn{2}{|c|}{$4.69 \pm 0.58$} & \multicolumn{2}{|c|}{100.5} & -1.49 & 0.151 & 0.259 \\
\hline Q03 & \multicolumn{2}{|c|}{$3.71 \pm 0.75$} & \multicolumn{2}{|c|}{$3.56 \pm 0.70$} & \multicolumn{2}{|c|}{150.5} & 0.57 & 0.596 & 0.100 \\
\hline Q04 & \multicolumn{2}{|c|}{$3.00 \pm 0.00$} & \multicolumn{2}{|c|}{$2.94 \pm 0.24$} & \multicolumn{2}{|c|}{144.5} & 1.03 & 0.485 & 0.179 \\
\hline Q05 & \multicolumn{2}{|c|}{$3.24 \pm 0.81$} & \multicolumn{2}{|c|}{$3.44 \pm 0.86$} & \multicolumn{2}{|c|}{121.5} & -0.58 & 0.574 & 0.101 \\
\hline Q06 & \multicolumn{2}{|c|}{$3.88 \pm 0.58$} & \multicolumn{2}{|c|}{$4.00 \pm 0.50$} & \multicolumn{2}{|c|}{122.0} & -0.62 & 0.661 & 0.109 \\
\hline Q07 & \multicolumn{2}{|c|}{$3.71 \pm 0.67$} & \multicolumn{2}{|c|}{$3.38 \pm 0.70$} & \multicolumn{2}{|c|}{163.0} & 1.07 & 0.287 & 0.186 \\
\hline Q08 & \multicolumn{2}{|c|}{$4.12 \pm 0.68$} & \multicolumn{2}{|c|}{$4.00 \pm 0.71$} & \multicolumn{2}{|c|}{148.0} & 0.47 & 0.773 & 0.082 \\
\hline Q09 & \multicolumn{2}{|c|}{$4.41 \pm 0.60$} & \multicolumn{2}{|c|}{$4.69 \pm 0.58$} & \multicolumn{2}{|c|}{100.5} & -1.49 & 0.151 & 0.259 \\
\hline Q10 & \multicolumn{2}{|c|}{$3.71 \pm 0.75$} & \multicolumn{2}{|c|}{$3.56 \pm 0.70$} & & & 0.57 & 0.596 & 0.100 \\
\hline Q11 & $3.00 \pm 0.00$ & & $2.94 \pm 0$ & & & & 1.03 & 0.485 & 0.179 \\
\hline$\overline{\mathrm{Ma}}$ & & & & & & " & Id Satis & ion. GA & $\begin{array}{l}\text { Group } \\
\text { d first). }\end{array}$ \\
\hline & 1月DLL1 & & $20 \pi$ & & & & 30 & NS & \\
\hline \# & $\begin{array}{c}\text { Mode } \\
\text { GA }\end{array}$ & & de GB & & & $\mathrm{df}$ & $\mathrm{N}$ & $\mathrm{p}$ & $\begin{array}{l}\text { Cram } \\
\text { er's V }\end{array}$ \\
\hline Q12 & Neutral & & -world & & & 1 & 32 & 0.297 & 0.184 \\
\hline Q13 & Neutral & & tral & & & 1 & 31 & 0.795 & 0.047 \\
\hline Q14 & Neutral & & -world & & & 1 & 27 & 0.179 & 0.259 \\
\hline Q15 & Neutral & & -world & & & 1 & 26 & 0.257 & 0.222 \\
\hline Q16 & Neutral & & -world & & & 1 & 32 & 0.273 & 0.194 \\
\hline Q17 & Neutral & & tral & & & 1 & 28 & 0.678 & 0.167 \\
\hline Q18 & Neutral & & tral & & & 3 & 30 & 0.875 & 0.094 \\
\hline Q19 & Neutral & & -world & & & 3 & 30 & 0.071 & 0.420 \\
\hline Q20 & Neutral & & tral & & & 3 & 29 & 0.682 & 0.228 \\
\hline
\end{tabular}

B. The mode of each question has also been included.

significant difference for the acquired knowledge (initial: $420.62 \pm 134.65)$, (after playing: $526.26 \pm 84.07)$, $(\mathrm{t}[15]=-$ $\left.2.80, \mathrm{p}=0.014^{* *}, \mathrm{~d}=0.70\right)$. For Group $\mathrm{B}$, a paired t-test revealed that there was also a statistically significant difference for the acquired knowledge, (initial: $380.88 \pm$ 
168.8), (after playing: $550.00 \pm 103.26),(\mathrm{t}[16]=-4.21$, $\left.\mathrm{p}<0.001^{* *}, \mathrm{~d}=1.02\right)$. These results indicate that the students improved their knowledge about teeth morphology with the two modes. We also checked if there were statistically significant differences in acquired knowledge between the two groups, Group A (526.26 \pm 84.07), Group B (550.00 \pm 103.26), $(\mathrm{t}[31]=-0.69, \mathrm{p}=0.495, \mathrm{~d}=-0.24)$. The $\mathrm{t}$-test indicates that no statistically significant difference was found with regard to the acquired knowledge using the two modes.

\section{B. Usability, Depth perception, and Satisfaction Outcomes}

Several non-parametric tests were performed for the Likert questions (the Mann-Whitney $U$ test for unpaired questions and the Wilcoxon Signed-rank sum test for paired questions) to determine if there were statistically significant differences in the opinions of the students depending on the game mode played. We applied tests to: 1) the answers to the Q2 questionnaire of the students that played the real-world version first versus the students that played the neutral version first; 2) the answers to the Q2 questionnaire versus the answers to the Q2 questionnaire of the students that played the real-world version first; 3) the answers to the Q2 questionnaire versus the answers to the answers to the Q2 questionnaire of the students that played the neutral version first; 4) the answers to the Q3 questionnaire of the students that played the real-world version first versus the students that played the neutral version first. No statistically significant differences were found in any of these tests. As an example, we only present the Mann-Whitney $U$ test for the first mentioned analysis, Q2 for GA vs. GB (Table II).

With regard to the 3D sensation (Q08-Q10), the students thought that the models looked like real plaster models (Q08: $4.48 \pm 1.58$ over 7$)$. The sensation that the teeth come out of the screen was considerable (Q09: $5.09 \pm 1.14$ over 7).

Finally, the last question asked was about rating the experience from 1 to 10 . The students liked the game experience (Q11: $8.03 \pm 1.31$ over 10). Four professors also tested the game; they liked the game and they argued about the great possibilities of including this game in their classes. The professors expressed their satisfaction with the game and scored it with $8.20 \pm 1.79$. Most of them preferred the neutral-background version (75\%).

\section{Preferences about the Game Background}

To measure the students' preferences about the two modes, several chi-squared $(\chi 2)$ tests were performed. These tests show whether a group has a preference for the game mode. Table III shows the modes of questions and the result of the analysis. The $\chi 2$ test revealed that there was no statistically significant difference between the two different backgrounds. However, if percentages are considered, the following was observed. When asked about the best option for dental learning (Q213), 68\% preferred the neutral background; $63 \%$ considered the neutral background to be easier to manipulate (Q214); 58\% considered the neutral background to be more comfortable (Q215); 64\% considered that the images/models looked better in the neutral background (Q17). With regard to their recommendation for using the two versions at school (Q20), their preferences were the following: 55\% selected the neutral background, $24 \%$ selected both, $14 \%$ selected the real-world background, and $7 \%$ selected neither of them. A total of $53 \%$ of the participants liked the real-world background the most (Q12). For the most fun mode (Q19), 43\% selected the real-world background, $37 \%$ selected both, and $20 \%$ selected the neutral background. From these percentages and the results of the $\chi^{2}$ test, we can deduce that although there were no statistically significant erences betwee ie two sdes, the dental students expressed their preference for the neutral background.

\section{DISCUSSION}

The first of our hypotheses was that the students would increase tl know ge ab teeth orphology. We compared their initial knowledge and their knowledge after playing. We found statistically significant differences that corroborate the first hypothesis $(\mathrm{p}<0.05)$. Therefore, our results indicate that our game improves students' learning performance. This result is in line with previous conclusions that have argued for the great potential of educational computer games in helping students to improve their learning performance [17]. However, the second hypothesis (the real-world background will obtain better learning results) was not corroborated. Although unexpected, it is an excellent result because it means that the game is well suited for learning outcomes and that the two backgrounds can be used for this purpose.

For preferences regarding the background mode, even though there were no statically significant differences between the two modes the percentages and the results of the $\chi 2$ test indicated that, the dental students preferred the neutral background for learning activities (Table III). For Q13 ("Which game is better for dental learning?"), the preferred option of the two groups was the neutral background. The same occurred for Q18 ("Which of the games would you recommend to your classmates") and Q20 ("Which game do you want your teacher to use in class?"). Two of the students' arguments that support this preference are: "The real world in the game distracted me" and "With the neutral background, I could focus on the morphology of the teeth".

For the experienced fun, the students had fun playing the game (means of 4 on a scale from 1 to 5 ). Even though there were no statistically significant differences between the two backgrounds, the students assigned higher means to the realworld background. In related questions shown in Table III, the preferred background mode for Group B was the realworld background (Q19 and Q12). These data, the opinion of the professors, and the comments of the students indicate that the real-world background was identified as being more suitable for leisure activities. One of the students' comments that support this argument is: "You can see yourself inside the TV and it is entertaining". 
For depth perception, the results showed that the 3D sensation (Q09) was greatly perceived and appreciated. This result is in line with a similar question answered by children in the study of Arino et al. [11]. Our results also revealed that autostereoscopy gave the students the feeling of being able to touch the 3D elements (Q10). These results are encouraging and can be exploited for the development of educational systems.

With regard to the time spent placing each tooth in its correct position, the students spent more time on some teeth whose morphology was difficult to distinguish. The opinion of the professors who participated in the study was that the game facilitates the correct identification of these teeth and that this identification is much more difficult to do using traditional methodologies. However, this aspect was not analyzed in our study.

Although the results are encouraging, the use of this technology in the classroom presents several drawbacks. First, there is the cost of the autostereoscopic display, which is more expensive than normal displays. However, cheaper 3D technologies could be used. Second, the use of Kinect for the interaction limits the number of students that can be behind the student who is using the game.

\section{CONCLUSIONS}

In this paper, autostereoscopy and Natural User Interfaces were used to develop a serious game with two background modes for dentistry students. The two different modes were developed for an educational game based on teeth morphology. The game allows the users to have a complete experience without having to carry devices or wires on their bodies. To our knowledge, this is the first time that a game of these characteristics has been presented for learning dentistry. Based on our study, we believe that using natural gesture interaction and having stereoscopic vision without wearing devices or wires provides an enhanced and richer user experience that is metaphorically similar to the real-world experience. In this situation, the selection of elements is done by using your hands and interacting directly without other devices. Moreover, according to our results, the neutral background is especially suitable for learning activities and the real-world background has been identified as being more suitable for leisure activities. With regard to future work, we have compared two modes, but other comparisons are also possible; for example, using a control group in which the students learn teeth morphology using traditional learning method. Another possible comparison could consider autostereoscopic vs. non-autostereoscopic visualizations.

\section{ACKNOWLEDGMENTS}

This work received financial support from the Conselleria d'Educació, Investigació, Cultura i Esport through the grant for consolidable research groups (20172018).
We would like to thank the following for their contributions: 1) The School of Dentistry C.F. FolgueraVicent (Alboraya, Spain); 2) Fernando Folguera, Agustín Herrero, Sandra Albinyana, and Juan-Fernando MartínSanJosé for their help; 3) The undergraduate students, the Master's students and the employees of C.F. FolgueraVicent who participated in the study; 4) The reviewers for their valuable comments.

\section{REFERENCES}

[1] K. Fishkin, "A taxonomy for and analysis of tangible interfaces," Pers. Ubiquitous Comput., vol. 8, no. 5, pp. 347-358, Jul. 2004.

[2] D. Roman and David, "Interact naturally," Commun. ACM, vol. 53, no. 6, p. 12, Jun. 2010.

[3] Y.-J. Chang, S.-F. Chen, and J.-D. Huang, "A Kinect-based system for physical rehabilitation: A pilot study for young adults with motor disabilities," Res. Dev. Disabil., vol. 32, no. 6, pp. 2566-2570, 2011.

[4] D. Rodríguez-Andrés, M.-C. Juan, M. Méndez-López, E. PérezHernández, and J. Lluch, "MnemoCity Task: Assessment of Childrens Spatial Memory Using Stereoscopy and Virtual Environments," PLoS One, vol. 11, no. 8, p. e0161858, Aug. 2016.

[5] Y.-J. Chang, S.-F. Chen, and A.-F. Chuang, "A gesture recognition system to transition autonomously through vocational tasks for individuals with cognitive impairments," Res. Dev. Disabil., vol. 32, no. 6, pp. 2064-2068, 2011.

[6] J. Anacleto, S. Fels, and R. Silvestre, "Transforming a Paper based Process to a Natural user Interfaces Process in a Chronic Care Hospital," Procedia Comput. Sci., vol. 14, pp. 173-180, 2012.

[7] M. Halle, "Autostereoscopic displays and computer graphics," SIGGRAPH, vol. 31, no. 2, pp. 58-62, 1997.

[8] D. J. Sandin, T. Margolis, J. Ge, J. Girado, T. Peterka, and T. A. DeFanti, "The Varrier TM autostereoscopic virtual reality display," in ACM SIGGRAPH 2005, vol. 24, no. 3, p. 894, 2005.

[9] A. Maimone, J. Bidwell, K. Peng, and H. Fuchs, "Enhanced personal autostereoscopic telepresence system using commodity depth cameras," Comput. Graph., vol. 36, pp. 791-807, 2012.

[10]H. Kim, G. A. Lee, U. Yang, T. Kwak, and K.-H. Kim, "Dual Autostereoscopic Display Platform for Multi-user Collaboration with Natural Interaction," ETRI J., vol. 34, no. 3, pp. 466-469, Jun. 2012.

[11]J.-J. Arino, M.-C. Juan, J.-A. Gil-Gómez, and R. Mollá, "A comparative study using an autostereoscopic display with augmented and virtual reality," Behav. Inf. Technol., vol. 33, no. 6, pp. 646-655, Aug. 2013.

[12] S. Meckfessel, C. Stühmer, K.-H. Bormann, T. Kupka, M. Behrends, H. Matthies, B. Vaske, M. Stiesch, N.-C. Gellrich, and M. Rücker, "Introduction of e-learning in dental radiology reveals significantly improved results in final examination.," J. Cranio-Maxillofacial Surg., vol. 39, no. 1, pp. 40-48, Jan. 2011.

[13]P. Grigg and C. D. Stephens, "Computer-assisted learning in dentistry a view from the UK," J. Dent., vol. 26, no. 5-6, pp. 387-395, Jul. 1998.

[14]D. Walmsley, "Computer-aided learning programmes in teaching dental students," Evid. Based. Dent., vol. 4, no. 4, pp. 81-81, 2003.

[15]A. Welk, C. Splieth, E. Wierinck, R. O. Gilpatrick, and G. Meyer, "Computer-assisted learning and simulation systems in dentistry--a challenge to society," Int. J. Comput. Dent., vol. 9, no. 3, pp. 253-253, 2006.

[16]H. Rosenberg, H. Grad, and D. Matear, "The effectiveness of computer-aided, self-instructional programs in dental education: a systematic review of the literature," J. Dent. Educ., vol. 67, no. 5, pp. 524-532, May 2003.

[17]G.-J. Hwang and P.-H. Wu, "Advancements and trends in digital game-based learning research: a review of publications in selected journals from 2001 to 2010," Br. J. Educ. Technol., vol. 43, no. 1, pp. E6-E10, Jan. 2012. 